\title{
Achieving Dialogue with Children with Severe Autism in an Adaptive Multisensory Interaction: the "MEDIATE" project
}

\author{
Narcís Parés, Paul Masri, Gerard van Wolferen, and Chris Creed
}

\begin{abstract}
This paper presents an adaptive physical environment that allows children with severe autism to successfully interact with multimodal stimuli, giving them a sense of control of the interaction and hence providing them with a sense of agency. This has been an extremely important effort for two main reasons: (a) this user group cannot be typified, hence making the design of an interactive system to fit all the spectrum of individuals a very complex task; (b) each individual PAS (Person on the Autistic Spectrum) user must be able to develop himself within the environment according to his own capacities and potentiality. Qualitative evaluation by psychologists shows very good results and sketches an encouraging future for research on these environments.
\end{abstract}

Index Terms-Artificial, augmented, and virtual realities, Assistive technologies for persons with disabilities, Interaction styles, Psychology, Metadata.

\section{INTRODUCTION}

$\mathrm{B}$ ECAUSE of the exceptional type of user involved, the design of interactive systems for children with severe autism and no verbal communication is one of the most challenging areas a designer of interactive systems can approach. In spite of previous attempts by other research projects to develop therapeutic and/or educational applications for people with autism (e.g. [1], [2], [3], [4], [5]), psychologists in our project consortium believe that our understanding of autism does not yet permit us to aim for such ambitious goals, especially in low functioning PAS (persons in the autistic spectrum). Hence, we would like to stress that MEDIATE does not claim to be either therapeutic, or educational at this point.

MEDIATE (A Multisensory Environment Design for an Interface between Autistic and Typical Expressiveness [6], [7]) is an adaptive environment that generates real time stimuli (visual, aural and vibrotactile) such that low functioning PAS children, who have no verbal communication, can express themselves and "have a bit of fun". This goal, in spite of its apparent simplicity, is actually quite demanding and ambitious, both in psychological and in technological terms.

In this paper we will describe the design approach, functionality and results of the environment whose adaptive nature allows the wide range of PAS users to successfully interact with the multimodal outputs.

- N. Parés is with the Experimentation on Interactive Communication group, Audiovisual Institute, Universitat Pompeu Fabra, Pg. Circumval lació, 8, 08003 Barcelona, Spain.E-mail:npares@iua.upf.es.

- P. Masri and C. Creed are with the Responsive Environments Centre, School of Art, Design and Media, University of Portsmouth, Winston Churchill Avenue, Portsmouth, PO1 2DJ, UK. E-mail:

paulmasri.mediate@worldwithoutwalls.co.ukEchris.creed@port.ac.uk.

- G. van Wolferen is with the Faculteit Kunst, Media and Technologie,

HKU, PO Box 2471, Oude Amersfoortseweg 131, Hilversum, 1200 CL,

The Netherlands E-mail: gerard.vanwolferen@kmt.hku.nl.
Because the incidence of autism seems to be much larger in men than women (in a ratio that some experts define as 1:3 or even 1:10 [8]), this paper will always refer to the user in the male form.

\section{Defining Autism}

Autism is a set of disorders in intercommunication and interrelation abilities that leads to an impairment of cognitive and emotional development. The essential characteristics of this disorder are the presence of an abnormal development in the following areas [9]:

1. Communication: Difficult or non-existent verbal communication. Difficulties in non-verbal communication.

2. Socialization: Severe difficulties in interpersonal relationship.

3. Imagination: There is a lack of imagination characterized by uncommon and repetitive game play.

This is externally manifested in a lack of affective expression, an apparent lack of empathy, an obsessive concentration on particular elements and, often, repetitive movements. The factors that determine autism have a biological cause and the disorder is manifested during the first thirty months of the child's life [8]. The three main characteristics mentioned above, make the child feel isolated and alien from the surrounding world.

There are huge differences among individuals that are placed at different levels on the wide spectrum that goes from low functioning to high functioning. Moreover, autism is often diagnosed only after psychologists have determined that the child cannot be included within any of the other known disabilities. MEDIATE is designed for children between 6 and 12 years of age who are low functioning PAS with no verbal communication abilities. 


\section{GOALS OF MEDIATE}

A main goal and two secondary goals were defined by the interdisciplinary consortium that was created for MEDIATE:

1. Main goal:

- For the children with autism to have fun and have the chance to play, explore and be creative in a controllable and safe space.

2. Secondary goals (completely subject to ethical considerations):

a. For the psychologists to better understand autism and the possible underlying communication mechanisms.

b. For the parents of the children with autism to find new qualities in their sons or daughters by seeing them play in this environment.

\section{Strategies to ReACH THESE GOALS}

The consortium defined four strategic principles for achieving the above goals:

\subsection{Give users a Sense of Agency}

One of the underlying objectives of MEDIATE was to provide the user with clear interaction dialogues that would hopefully give the children a sense of control of the system. If this were achieved then they would probably gain what is known as the sense of agency that makes them feel at ease. This comes from the fact that autistic children rarely experience a sense of control with respect to their surrounding world. Because of the multiple layers of stimuli in everyday life, they cannot understand why events around them occur, even in the cases when they themselves are causing these events. The sense of agency, defined as the consciousness of being able to exert control over their surrounding environment and obtaining a coherent response, is an essential goal to be able to encourage the children to express themselves within the environment.

\subsection{Enhance Non-Repetitive Actions}

Children with autism often tend towards repetitive activity such as rocking back and forth or flapping an arm or a hand. They tend to do so when they are feeling overwhelmed by the surrounding stimuli. Apparently these stereotypical movements help them isolate themselves from the environment and feel at ease and relaxed. They can also be very repetitive in their actions when they are obsessed by something they like doing and hence do it over and over in an overexcited manner. Both attitudes are considered undesirable by psychologists, because they isolate the child from the world. Therefore, the second principle behind the design of MEDIATE was to detect repetitive patterns in all the streams of sensed data from the user.

\subsection{Adapt to each Child}

Because of the wide spectrum of children with autism that could use the environment, the consortium considered it very important to make the system adapt to each child's needs and potential. Therefore it was important that the behavior of the system should be neither fixed nor follow a predefined path, but vary in response to the user and adapt to the specific traits or 'signature' of the user. The aim behind this was always to try and engage the user in dialogue - that is, creative, explorative behavior with an interplay between the user and the environment, such that the user's sense of agency is preserved and enhanced.

\subsection{High Functioning Children in Design Team}

To be able to determine whether the design decisions were on the right track, groups of high functioning children with autism, who can give verbal feedback, were incorporated in the design teams, at each participating university, as informers. They gave useful comments on the type of stimuli being designed and the type of interactions proposed. For example, in Portsmouth an Asperger child gave good insight on vibration stimuli design and in a final stage helped in testing the robustness (both software and hardware) of the environment. Also, in Barcelona two high functioning boys and a girl helped in finding the most contingent interactions with visuals.

\section{Designing AN ENVIRONMENT}

MEDIATE presents a unique approach in that it centers its interest on interaction per se. Because the goal of giving low functioning PAS children a place to have a fun and relaxed experience is based on the hypothesis that it will be achieved by giving them a sense of control, interaction design becomes central.

\subsection{Stimuli}

Because interaction is so fundamental, it means that content related to interaction is not meaningful in MEDIATE and therefore the types and formats of stimuli chosen as output for the user's actions are basically abstract. Had we used representational stimuli, they would have been the arbitrary choices of the design team, which may or may not appeal to different users. Furthermore, the appeal or dislike of this content could possibly generate adverse reactions towards the project.

An example to illustrate this reasoning, in the area of visuals, would be the following. Let us imagine the visuals present the image of a dog or a virtual dog. This element will probably be interpreted contentwise by the user and may generate either a rejection (because of fear) or overexcitement (because of a special liking). In either case, there is a high probability that the user will focus on that specific content and present incapacity to perceive anything else in the environment.

Therefore, because we wanted the user to concentrate on the dialogue between him and the system, we wanted to put the accent on the proposed interaction and not on any content that could interfere with this interaction.

We believe that this is a novel approach. Indeed, all interactive and virtual reality applications used in disabilities, that we are aware of, focus on simulations of physical environments. For example, applications for treating psychological disorders such as phobias [10][11][12], ObsessiveCompulsive Disorders [10], Post-Traumatic Disorders 
[10][13], Attention Deficit Disorders [10] and Autism [1][2][14], are basically designed with specific content in mind: that of a place and/or situation to be simulated to expose the patient to achieve desensitation or training. Hence, the output stimuli are representational, whereas in MEDIATE, they are essentially abstract.

Of course the choice of the actual abstract stimuli was based on several criteria so that they would be appropriate for the interaction design. A detailed description of this design falls outside the scope of this paper, but for the case of visual stimuli in MEDIATE, Parés et al. presented it in [15].

The only work that we have found that places the emphasis on interaction in a similar way to MEDIATE is that by Tamar Weis et al. [16][17]. They are leisure experiences for people with cerebral palsy or stroke based on single camera 2D artificial vision systems, but the visuals are still representational.

\subsection{A Space for Interacting}

In order to give a sense of control to the low functioning children we had to design a system that would attempt to start a dialogue based on the actions of the user and respond with very contingent reactions. The actions of the user had to be necessarily very simple and almost without a specific goal behind them. Hence, we thought that full body interaction would provide such actions; i.e. moving and gesticulating.

But the idea was to impose as few restrictions as possible to the user and provide him with the most natural interaction approach possible. This would hopefully facilitate entering the environment in a relaxed attitude and spontaneously start the experience. Thus, MEDIATE was envisioned since its conception as an interactive physical environment that would allow the users to be in a free-roaming space, without invading the user's body or encumbering him with cables or sensors. With these constraints, our team faced a very important challenge: that of making the experience completely non-invasive. All sensors had to be placed in the environment looking towards the user, rather than placing them on the user himself. This is also a unique approach that no other interactive application or system has developed for PAS in the past.

Some past projects have undertaken the classical virtual reality approach, for example Strickland [1], [2]. This approach is extremely invasive for users and although it seems it was successful, it was only tested with two children and it remains unproven that most low functioning children will accept wearing a head-mounted display. At the same time the child is not truly free to move about and the interface seems very unnatural. Others have undertaken the desktop VR approach, for example in social skills training [18] or in supporting imagination [14], but these applications are mainly for high functioning users and they require the attention of the user to be focused on the screen for a long time, which is difficult for low functioning children. Also, the use of a joystick requires good motor control, which is very often beyond the abilities of low functioning PAS.

Other approaches have used robots, both fixed on a table or free-moving on the floor of a room; for example the work by Dautenhahn [4][19][20][21]. The psychologists of the consortium thought that for MEDIATE this "objectual" approach to interaction (as opposed to a space-based approach) could very easily lead the child to one of two possible and undesirable attitudes. On the one hand, the user could very easily lose interest in the object because of his inability to focus attention on an object for a long time, especially because in MEDIATE we were looking for a free individual interaction. On the other hand, the object could generate obsessive behaviors of the child on the object itself such that the child could completely forget about interaction. Another disadvantage of an objectual approach was the difficulty of defining and developing multimodal adaptive interaction on such a small element. Finally, interacting with an object could make external observation very difficult for the raters to be able to establish whether the child was gaining the desired sense of control and sense of agency.

\section{THE PHYSICAL ENVIRONMENT}

MEDIATE is a hexagonal space, approximately six meters in diameter (Fig. 1). It was designed to be large enough to promote movement of the user within it, but not so large so that the child would feel lost. The shape was chosen such that no sharp corners would create narrow spaces for the child to hide in. The space had to allow for full body interaction.

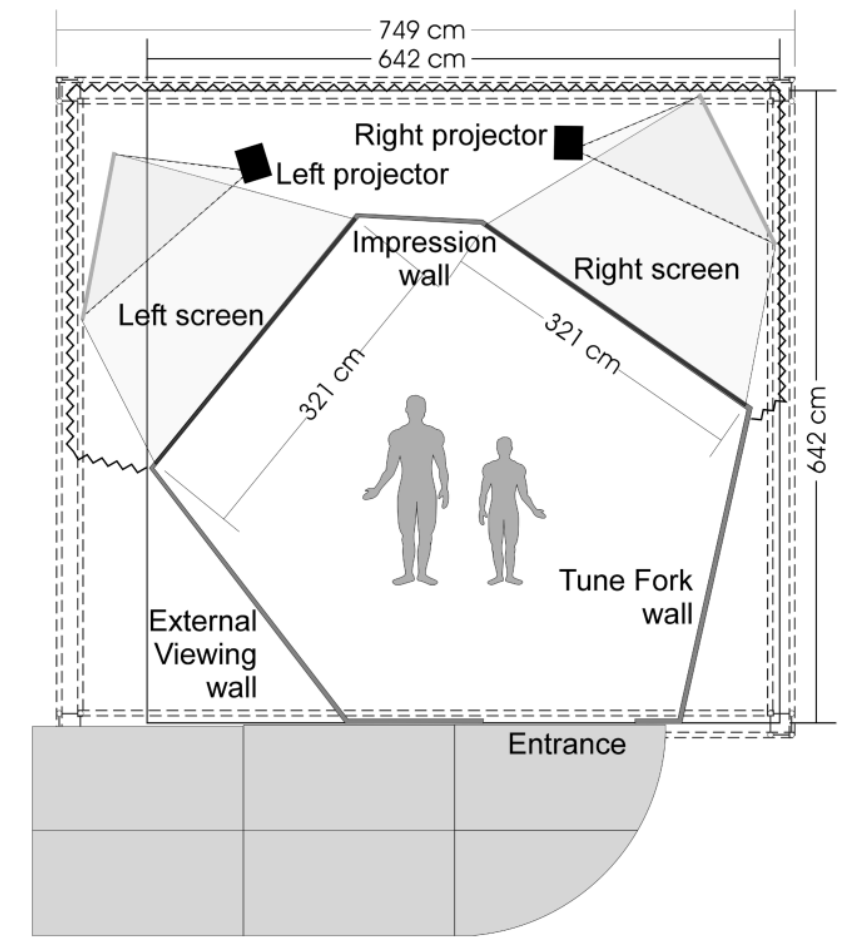

Fig. 1. Plan view of the MEDIATE physical environment. An external lightweight trilite structure encloses the internal hexagonal interaction space. 


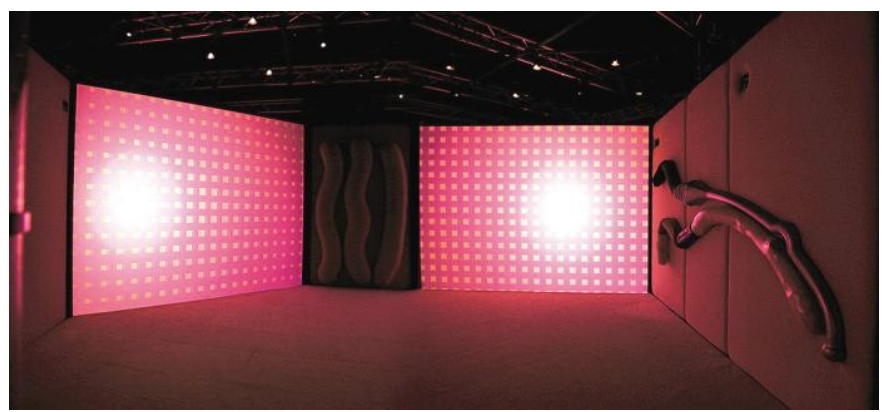

Fig. 2. Panoramic view of the interior of the environment with the interaction elements (anticlockwise from the bottom): interactive floor surface, tune fork wall, screen one, impression wall \& screen two.

Inside the space several elements act as interaction interfaces (Fig. 2). We briefly describe them in the following subsections.

\subsection{The Floor Surface}

It is a grid of wooden tiles (covered by a carpet) that can sense the impact of footsteps and also gradual shifting of weight. This is achieved by a tile module that has three layers: a bottom wood support, a top floating wood panel and a central element based on a piezo-ceramic pick-up sandwiched between two layers of industrial scouring fabric. Therefore, the data from these tiles is captured both as analogue 'crunch' and MIDI trigger. Further detail may be found in [22].

\subsection{The Tune Fork}

A wall with tube-like structures and a variety of textures that sense both, soft tactile input such as caressing or stroking, and sudden sharp impact such as tapping or drumming (Fig. 3). This structure contains several embedded piezo bi-morph sensors that capture the vibrations created by the user when caressing, tapping, etc., and are fed to MIDI triggers [22].

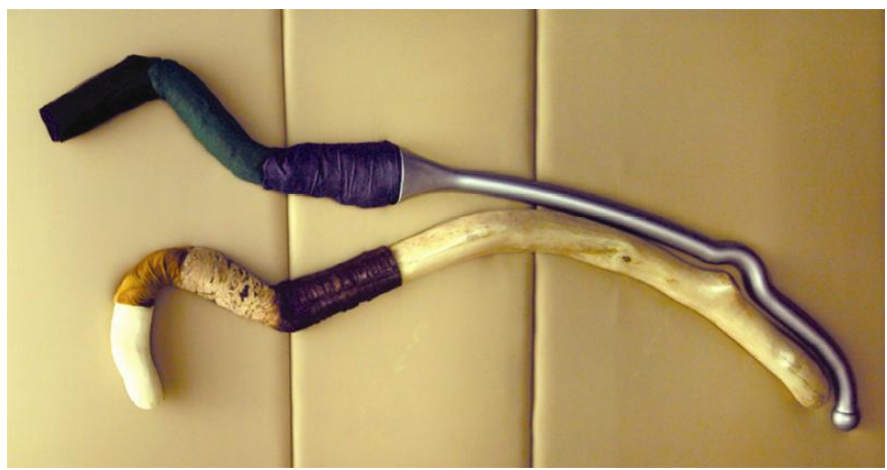

Fig. 3. Detailed view of the Tune Fork wall with its different types of textures and materials: e.g. cork, wood, rubber, metal, plastic, felt, etc.

\subsection{The Impression Wall}

A wall with padded structures, that senses pressure and can emit vibration (Fig. 4). Underneath the special padding made of medical foam, pressure gauges connected to an analog to digital control box capture the pressure exerted by the user in several points uniformly distributed in the structures. The floor, the Tune Fork and the Impression wall are all managed from a dedicated computer connected to the MIDI intermediary hardware [22].

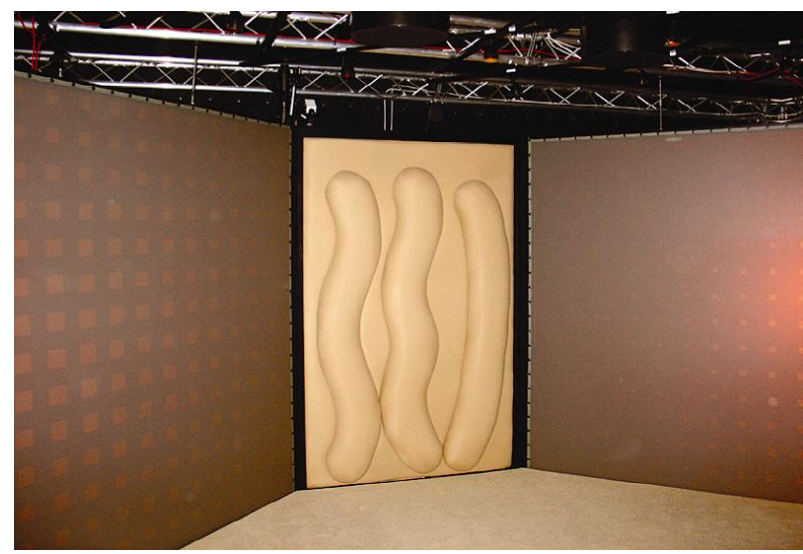

Fig. 4. Detailed view of the Impression wall (between the two screens), which is formed by three padded structures.

\subsection{Visual Interaction Walls}

Large rear projection screens $(300 \times 225 \mathrm{~cm})$ are used for graphical displays (Fig. 5) as well as giant touch and proximity screens. Visual interaction is based on a non-standard use of particle systems that allow for an abstract interaction that is very contingent and clear to give a good sense of control. A dedicated graphics workstation generates all visual interaction with a double head graphics board that sends the images for each screen to the corresponding projector. Further detail may be found in [15].

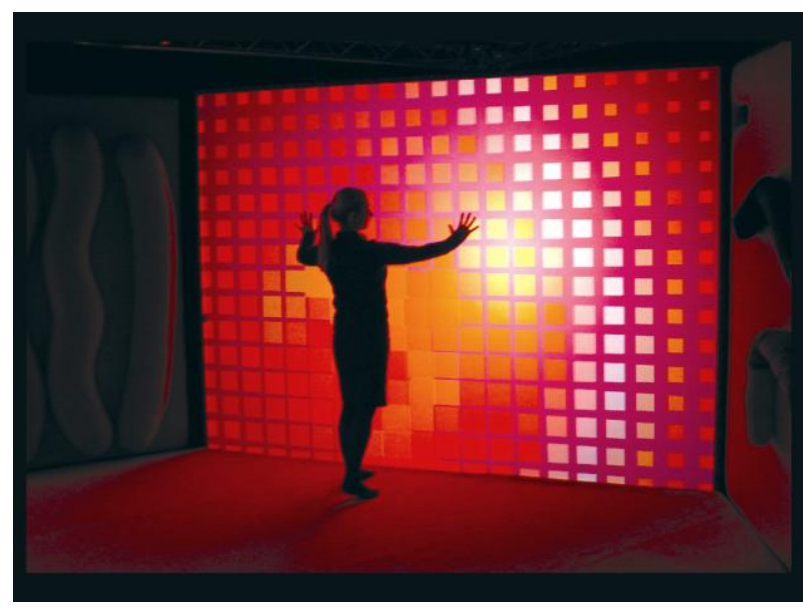

Fig. 5. A user (neurotypical adult) interacting with visuals at one of the touch sensitive, rear projection screens. The user is generating waves of color on the virtual tiles that fill the screen.

\subsection{Artificial Vision Sensing System}

There is a $2 \mathrm{D}$ artificial vision system based on near infrared cameras and lighting: seven cameras give coverage of the entire space enabling position tracking (with 3 cameras) as well as 2D identification and tracking of silhouette and limbs (with 4 cameras); in addition two other cameras, one 
behind each Screen wall, are used to simulate touch through proximity sensing. This system is managed by two dedicated computers with a total of three special video capture boards. For a complete description of this system please refer to [23].

\subsection{Sound Capture System}

A set of microphones distributed around the environment to sense sounds emitted by the child such as vocalizations and clapping. The sound system is controlled by a dedicated computer that uses special hardware to interface to the microphones. A full description may be found in [22].

\subsection{Sound Generation System}

A set of loudspeakers distributed around the environment enable MEDIATE to produce sound and control its localization. Sound is generated from a dedicated computer [22].

\subsection{The Entryway}

The visual sensors also provide a 'user is present' signal to the rest of the system and the environment responds to the user entering by 'waking up' from an ambient state to a live-response state and does the reverse as the user exits [15][23].

\subsection{Linking and managing subsystems}

All sensing and stimuli generation subsystems are linked by a $1 \mathrm{~Gb}$ Ethernet network to two computers that manage the "brain" of the system: the Signature Analyzer and the Decision Maker (explained below). The system is therefore completely controlled by eight computers, interconnected by a very fast network, that use a great deal of auxiliary hardware that is specific for each type of sensed or generated data. If MEDIATE, as it seems, were finally considered to be an interesting system for special education or care centers, it would be desirable to achieve a much greater integration of the system's hardware to make its control and management more accessible to non-technical personnel.

\section{Establishing the Sense of Agency}

When a user first enters the MEDIATE environment, the system response is at its simplest and most literal. The screens display a representation of the user that is analogous to a silhouette. It directly mimics the user's shape and movements in an immediate and obvious way. Similarly, sounds produced by the user, such as footsteps, are presented back to the user amplified and direct pressure exerted by the user on the Impression Wall results in a proportional vibratory response.

The correlation between the user's actions and the system's responses is simple and obvious. Unlike the world at large that contains many stimuli, only a few of which are in response to an individual, the MEDIATE environment provides a space where the stimuli are limited, focused and exclusively responsive to the user.

In this way, the system seeks to establish a dialogue with the user. The user may drive the response from the system through the way he chooses to move, make sound or reach out and touch it. But like any true dialogue, the interaction is guided by both participants in concert with one another. MEDIATE is not a passive partner and so, following the initial period when it allows the user to become accustomed to the environment, it begins to adapt its behavior.

\section{Achieving Dialogue}

For there to be dialogue between the user and MEDIATE, the environment must provide both an immediate response to user actions and also adapt its behavior over time. Our solution to this has been to separate the 'immediate response functions' from the 'behavior adaptation functions' and to handle them in distinct but connected modules (Fig. $6)$.

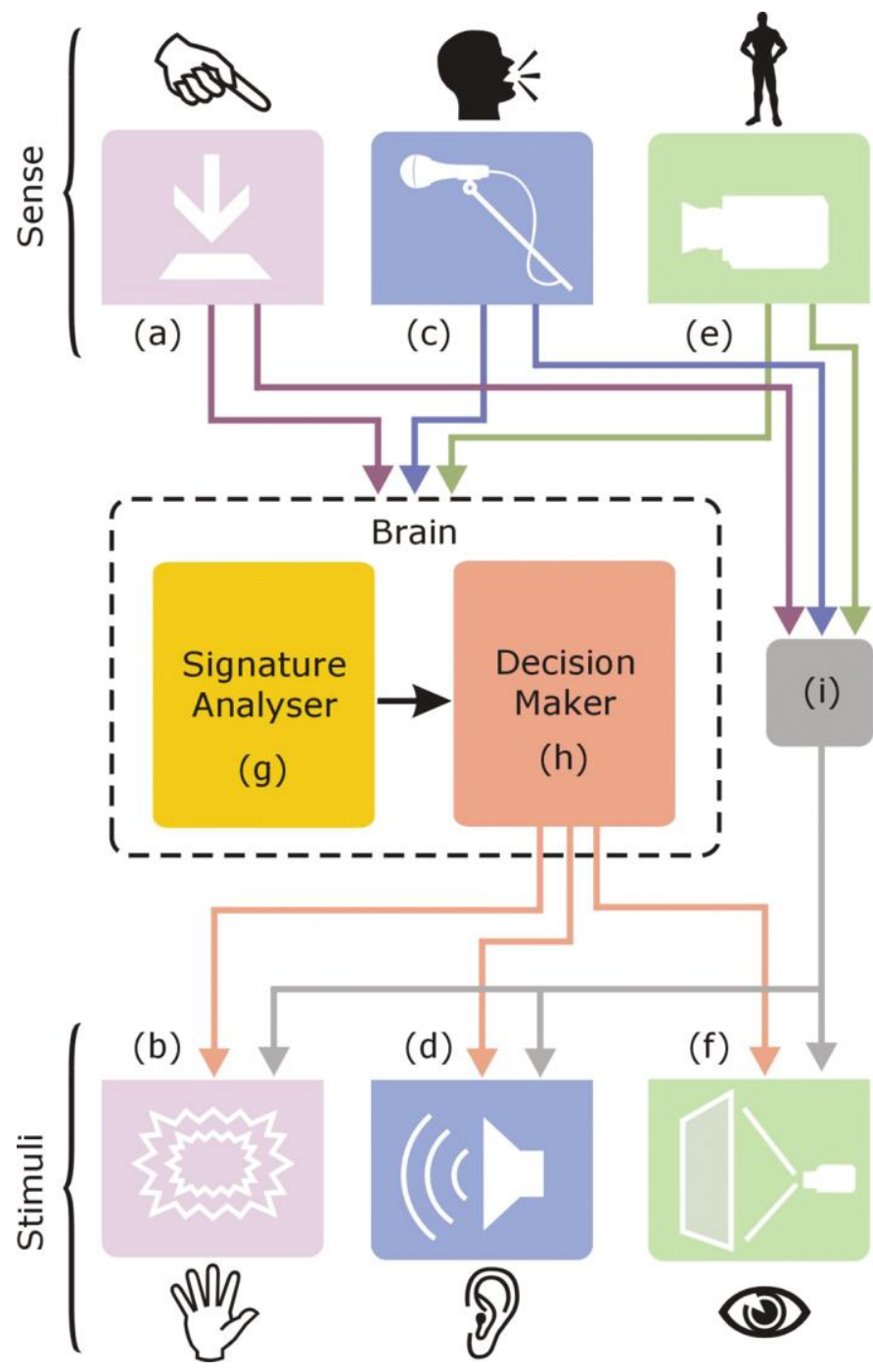

Fig. 6. Block diagram of the modules of the system: (a) touch sense, (b) vibration stimulus, (c) sound sense, (d) sound stimulus, (e) vision sense (silhouette, position, etc.), (f) vision stimulus, (g) Signature analyzer, (h) Decision Maker and (i) direct connection from senses to stimuli allowing for cross-modality.

Each of the system's stimuli (vibration, sounds and visuals) is driven by their own response module (Fig. 6.b, $d \&$ f). These incorporate sophisticated sense-stimulus mappings that are both flexible and controllable [15][22]. Their responses range from immediate mimicry to mediated, artis- 
tic interpretation, from self-modality (e.g. user makes a sound, so MEDIATE responds with a sound) to crossmodality (e.g. user's touch elicits a visual response). These axes of complexity are parameterized and are under the control of the "brain" of the system.

This "brain" is composed of a Signature Analyzer (Fig. 6.g) that looks for and reports on repetitive traits in the user's behavior and a Decision Maker (Fig. 6.h) that observes trends in the user's behavior and adapts the system behavior in response.

\section{Detecting Repetitive Behavior - The SIGNATURE ANALYZER}

Since repetitive behavior by a user is considered undesirable (as stated in section 4.2), the system must recognize such occurrences in order to change the dynamic of the interaction between user and system. It is the role of the Signature Analyzer to observe the user's actions, to build up a profile and to provide a continuous assessment of the user's repetitive behavior.

It scans the user's actions for personal traits and builds a profile - the user's 'signature' - that it represents as metadata. The analysis is performed in real-time and logged to file so that it can be used both during sessions by MEDIATE itself to modify its response and also after sessions for offline analysis by researchers.

Fundamental to this approach is the fact that the first time the user makes a particular gesture or sound, this gesture or sound will be stored as noise. The second time the same sequence appears it will be detected and identified as a pattern and in the third and further occurrences, it will be recognized. In this way Signature Analyzer builds what can be seen as a representation of the user's behavioral repertoire.

This is based on the "Structural Information Theory" by Frank Restle [24] and the "Curve Theory" by Theo Loevendie [25], who noticed that what we recognize in music is not what we hear in music, but rather what we recognize is the structure of what we hear. Our team interpreted this idea not as a characteristic for music only but as a characteristic for perception of any time-dependent action or activity in all of the tactile, visual and auditory domains.

The three sensing modalities (tactile, sonic and visual) (Fig. 6.a, c, e) process the user's actions and pass the results to the Signature Analyzer (Fig. 6.g), which builds as many databases as there are streams of sensor data. The most frequently occurring pattern, the longest pattern and the pattern with the highest impact (a numerical relation of both) are continuously assessed and made available to the MEDIATE system throughout a user session.

If the user walks around in circles, this will be sensed as a sequence of footfalls from the sensors in the floor tiles and also as a series of motion paths by the camera system. Signature Analyzer detects the circular motion because it can observe multiple occurrences of the same (or similar) data sequences. In other words, it has no knowledge of circles per se, but it can detect repeated circular motion because the sensor data contains recognizable patterns.

Furthermore, Signature Analyzer is able to distinguish meaningful patterns from noise and jitter, detect similarity and not just identity, and include temporal, spatial and rotational invariance where appropriate. This is achieved respectively by placing upper and lower time limits and pattern length limits, by reducing actual sensor data to a series of 'primitives' and by processing or combining actual sensor data streams to create new virtual sensor data streams.

\section{Controlling System Behavior - The DECISION MAKER}

Decision Maker's role (Fig. 6.h) is to modify the overall behavior of the MEDIATE system with the goal of establishing, maintaining and developing dialogue with the user. In broad terms it does this by modeling the user's behavior and applying rules based on current user behavior, current system state and historical trends of both the user and the system.

In order to analyze the user's behavior, Decision Maker takes a holistic view of the user's actions. It is neither concerned with the low-level detail of specific sensors, nor with short-term (millisecond) fluctuations. Instead it focuses on abstracted metadata that inform it about shifts in the user's activity and repetition levels and where that activity and repetition has been directed (in the broad terms of the visual, sonic and tactile modalities). In turn, it does not direct the moment-to-moment responses of the system, but affects them indirectly and gradually by altering the control parameters of the stimulus modules. Because behavioral changes happen over several seconds, there was no need for rapid, continuous control - we found that running the Decision Maker process twice per second was more than adequate to achieve smooth changes.

The approach we chose for modeling, tracking and controlling within Decision Maker was to use a combination of simple components whose behavior is well known and easy to adjust and to assemble them in such a way as to create a powerful control system, rather than employ a more complex single control unit. The components are a state machine, 1st \& 2nd order moving average filters, threshold comparators and a simple set of heuristics; yet the end result is sophisticated and easy to tune.

\subsection{The User Behavior Model}

The user's behavior is modeled using a set of meta-sensors that track levels of activity and repetition.

Data from all the sensors is individually pre-processed to generate a set of normalized activity values. A moving timeaverage is applied to smooth out momentary spikes and noise and the data is collated by modality to give a running indication of activity levels for each modality. Using a similar approach, repetitions of the most significant detected patterns (provided by Signature Analyzer) are individually tracked over time using a bank of moving average filters, and then combined by modality to produce overall repetition levels.

In addition to the three activity meters and the three repetition meters, there are two derived meters, one for sustained inactivity (which grows when the global activity level remains low for a period of time) and one for sustained repetition (which grows when then the global repetition level remains high for a period of time). Together, these eight parameters are used to model the user's behavior. 


\subsection{The System Behavior Model}

The behavior of the system is set by adjusting the control parameters of the stimulus modules. In essence, each stimulus module is instructed which sensor data it should respond to and the level of complexity it should use for its sense-stimulus response functions.

Four sense-to-stimulus mappings were defined that apply to all modalities. These were ordered by their immediacy and directness in relation to the user's action. Using musical terminology, they were named Imitate, Echo, Comment and Counterpoint. In each mapping the stimulus is a response to the user's actions, but Counterpoint produces a more 'mediated', loosely-coupled response than Imitate, which directly mimics or amplifies the user's actions.

Within each mapping, the complexity can be modulated using the Richness parameter. This affects the perceived intensity of the mapping and provides for more subtle variations than mapping changes.

Mappings and Richness represent one axis of complexity. The other is how the modalities inter-relate. Selfmodality mappings (e.g. visual sense to visual response) are the simplest and cross-modality mappings (e.g. tactile sense to sonic response) are more complex (Fig. 6.i).

At the simplest level, indeed the state in which a session begins, each stimulus module responds only to sense data for its self-modality mapping and that is set to Imitate (mapping \#1) with low richness. At the most complex, it would be theoretically possible for all stimuli to be responding to all sense data with a combination of mappings and richness. In reality, because Decision Maker evolves the system complexity gradually and tailors the response to the user, the combinations tend to be much simpler. And the user, through his response is able to exert control over the complexity that he is willing to explore. Nevertheless, the breadth of the potential palette is such that it is possible for significant variation between sessions.

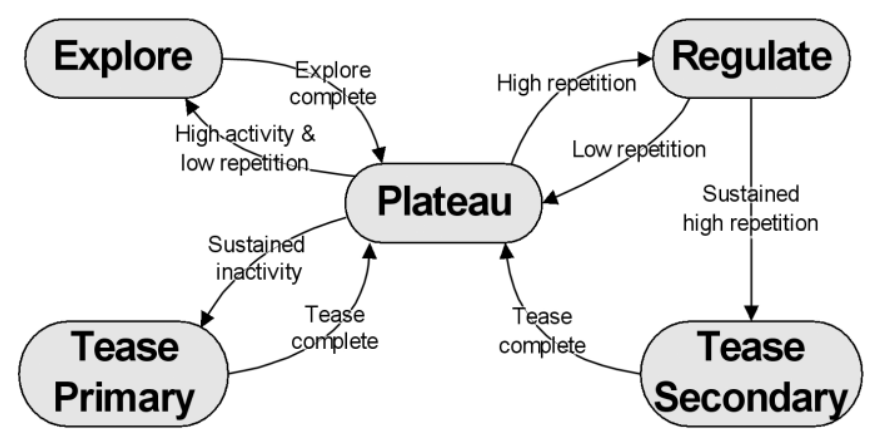

Fig. 7. Decision Maker state machine: states and state transitions.

\subsection{The System Internal State}

The Decision Maker state-machine has the following five states (Fig. 7):

Plateau: This is the simplest state, whose purpose is to preserve the status quo. It is used in the initial phase of each session to enable the user to become familiar with the environment, immediately after significant changes (such as a tease) and following a period of exploration (so that the user may experience and gain some familiarity with the new state without constant modifications). This state has a timer and the state cannot change until the timer has completed. The total elapsed time depends on the situation: for the initial phase it will be several minutes, whereas after a tease it may be only a few seconds.

Regulate: This state regulates the amount of complexity that the system exhibits. It is activated when the user displays significant repetitive behavior and its purpose is to reduce the complexity of the system response to a level that the user feels comfortable with and able to engage creatively with. It acts by 'dimming' the system response (in terms of complexity) when the user is behaving repetitively.

Explore: This state enables new elements to be introduced, such as higher order mappings and cross-modality interactions. It also implements some elements of the Regulate state, to ensure the system does not run away from the user. If Regulate can be thought of as vertical movement on the complexity scale, then Explore allows for sideways and upward movement. This state is activated when the user shows sustained activity that is non-repetitive (i.e. is creatively engaged).

Tease Primary: If the user is passive and unengaged (detected as sustained inactivity), the system activates a 'tease' that aims to grab the user's attention and re-engage him. The tease is an unsolicited stimulus from MEDIATE. 'Tease Primary' means that the tease comes from the user's 'primary modality', the modality that he was previously most active in.

Tease Secondary: If the user is active but has become obsessed with getting a particular response by repeating a gesture or vocalization over and over (detected as sustained repetition), the system activates a tease in a secondary modality - one that the user is not already engaged with. The aim is to distract the user from his obsession and gain his interest in a new mode of interaction. Typically, Tease Secondary is activated when the Regulate state has been engaged for some time simplifying the system response, without successfully encouraging the user out of their repetitive cycle of behavior.

Fig. 7 shows both the states and the ways in which MEDIATE can transition from one state to another.

\section{RefinIng MEDIATE}

The MEDIATE environment described in this paper is already the product of countless refinements, from alignment of physical devices and tuning of the sense-stimulus responses through to the timing and sensitivity of the Signature Analyzer and Decision Maker. It began with engineering solutions, progressed to investigating the interplay between different modalities and mappings and finished with refinements to the behavior. It has required development by individuals as well as team-wide discussions. The modifications have been prompted by technical issues, trial and observation, and has been greatly assisted by feedback from the 'informers' (high functioning PAS, see section 4.4) and the psychology team members.

In the process, it has been necessary to streamline the design, dropping some interesting, advanced or simply fun features in favor of pragmatism to get a fully functioning 
system. Also the design process and the user sessions have highlighted new features that would be desirable. The team aims to conduct a follow-on project that will investigate and incorporate some of these.

They include amongst others:

- making the system modular and scalable, so that it is possible to add or detach whole sense or stimulus interfaces and still have a meaningful, useful system;

- integrating the software into a single unit - currently multiple platforms, operating systems and development environments are used, with consequential complexities and vulnerabilities;

- redefining the 'modalities' in terms of how they are used - we discovered that the current segmentation by sense was ambiguous in certain circumstances since multiple modalities can be activated by a single action and conversely multiple actions may activate a single modality;

- extending the inter-modality interactions and widening the palette of mappings to create separate 'scenes' that may encourage creativity in different ways.

- to enable the patterns, found by Signature Analyzer, in the child's use of one modality to structure the system's feedback in another modality.

\section{Results}

MEDIATE is a transportable environment and sessions with low functioning children with autism have been held in London during two weeks, in Hilversum (Netherlands) for five weeks, in Barcelona for four weeks and in Portsmouth for six more weeks. More than 120 sessions corresponding to more than 90 low functioning PAS children have been held in total.

The earlier sessions were primarily used by the project team for system refinement and tuning with the target audience. Nevertheless, results from these early sessions as interpreted to us by the children's parents and carers were that most children entered the environment without effort and only one has been reported to refuse to enter on a first session. Needless to say, the children are not forced to enter in any way. In fact, the psychologists ask the parents (who are present during interaction just outside the action space) not to push (either physically or verbally) their children to enter the environment. This is already a huge success for the environment. These children, who need very rigid daily routines and who do not cope well with unknown places, have actually become curious enough to enter by their own will and start playing. The time spent in the environment has varied from 5 minutes to 35 minutes. In every case, it has been clear that the children have found at least one of the proposed interactions and have successfully played with it.

With the later sessions in Portsmouth no further environment changes and tunings took place so that more systematic assessments might be made. Video recordings were made and rated for agency by psychologists who had not been involved with the project. Further pre- and post- session tests for generativity were administered.

According to the psychological results, none of the children felt uneasy or uncomfortable in the environment (only one of the sessions had to be stopped because of overexcitement of the child) and most appeared to gain the desired sense of control and agency. No differences between the before and after session tests of 'creativity' were found. However, the numbers experiencing these systematic recording sessions are at present really too small for sensible statistical analysis (12 children, two sessions each). More data is being collected to increase these numbers.

On the other hand, feedback from parents and carers of this small group of children is illuminating. This feedback was obtained by an independent evaluator (appointed by The National Autistic Society, UK) who ran a four-hour group meeting with 9 of the parents of the Portsmouth group. The full report of this meeting is included in [26]. These parents felt that MEDIATE was a hugely beneficial experience that they would like to be able to continue to use. More specifically the feedback highlighted three main areas of benefit, these being ([26] pages 71-75):

1. Independence

2. Person-centered

3. No parental demands

In other words, the MEDIATE experience allowed many children for the first time to be completely on their own and be safe to make their own choices, enjoy their behaviors and get some recognition from the environment as to what they were doing. The environment allowed each individual child to do as much or as little as they wanted. Moreover, this was achieved taking careful consideration of their particular sensory needs and communication difficulties. Finally, in the MEDIATE environment, many of the children could do as they pleased without having to meet the expectations or demands of others, including their parents or carers.

\section{CONCLUSION}

Research using MEDIATE continues. Systematic data collection, as mentioned above is ongoing. Additionally a developmental programme involving a small group (five) of PAS children has started. This programme is run by the Portsmouth Education Authority Psychology Service. The children are preschool age and have specific social communication and interaction impairments. These children are receiving intensive (15 hours per week), structured activities with their own personal, trained carer throughout a 12month period. Weekly sessions in the MEDIATE environment for each child are one of these activities. Data about the environment's use, in the context of monitored changes in the children's general behavior over time, will provide valuable insight about additional richness and further tunings that may need to be added. Further we hope to gain information about the part the MEDIATE experience may play in these children's general development. Finally, we hope to gain information about the part the MEDIATE experience may play in these children's general development at which point it will be sensible to start to consider possible therapeutic/clinical uses of the environment. 


\section{ACKNOWLEDGMENT}

The authors wish to thank all the families and special education centers that have generously participated in MEDIATE. Also thanks to all the members of the teams of the project consortium who haven't signed this paper but had an essential part in making the project come true. Thanks are also due to The National Autistic Society, UK for organizing and running the evaluation study with the Portsmouth parents. Special thanks go to our high functioning informants who guided us in the early days of design.

MEDIATE is a project under the FP5 / IST / Systems and Services for the Citizen / Persons with special needs (including the elderly and the disabled) lasting for thirtysix months. It is coordinated by the "Responsive Environments Centre" of the "School of Art, Design and Media" of the University of Portsmouth (U.K.). The rest of the consortium is composed of: "Faculteit Kunst, Media and Technologie" of the Hogeschool voor de Kunsten Utrecht (Hilversum, Netherlands); the "Institute of Psychiatry" of Kings College London (U.K.); Show Connections Limited (Crowborough, U.K.) and the "Experimentation on Interactive Communication" group of the "Audiovisual Institute" of the Universitat Pompeu Fabra (Barcelona, Spain).

\section{REFERENCES}

[1] D. Strickland, "A Virtual Reality Application with Autistic Children," PRESENCE: Teleoperators and Virtual Environments. MIT Press, vol 5.3. pp. 319-329, 1996.

[2] D. Strickland, "Virtual Reality for the Treatment of Autism," Virtual Reality in Neuro-Psycho-Physiology, G. Riva, ed., Amsterdam, Netherlands: Ios Press, pp. 81-86, 1997, 1998.

[3] K. Dautenhahn, "Design issues on interactive environments for children with autism," Proc. 3rd Intl Conf. Disability, Virtual Reality \& Assoc. Tech., Alghero, Italy, pp. 153-159, 2000.

[4] K. Dautenhahn, Workshop on Robotic and Virtual Interactive Systems in Autism Therapy, A two-day workshop at University of Hertfordshire, Hatfield, UK. 27-28 September 2001. http:/ / homepages.feis.herts.ac.uk/ comqkd/ AutismWorkshop. htm (URL accessed Dec. 2004)

[5] J. Nadel and A. Revel, Robotic and Virtual Interactive Systems in Therapy of autism and other psychopathological disorders. A two-day international workshop at Hospital La Salpêtrière, Paris. 27-28 September 2002.

[6] MEDIATE official web site, URL:http://www.port.ac.uk/research/mediate/ , last accessed 27/02/05.

[7] P. Newland and C. Creed, "MEDIATE: Steps Towards a SelfOrganising Interface" Proc. of the Fifth International CAiiA Research Conference on Consciousness Reframed, R. Ascott, (ed), Newport, Wales: University of Wales, 2003.

[8] F. Happé, Autism, an introduction to psychological theory, Psychology Press Ltd, Taylor \& Francis Group, 1999.

[9] NAS, National Autistic Society UK, URL: http://www.nas.org.uk/, last accessed 27/02/05, 2005.

[10] M.M. North, S.M. North, and J.R. Coble, "Virtual reality therapy: An effective treatment for psychological disorders," In K.M. Stanney (ed), Handbook of Virtual Environments: Design, Implementation, and Applications. Mahwah, NJ: Lawrence Erlbaum, pp. 1065-1078, 2002.
[11] D.P. Pertaub, M. Slater, and C. Barker, "An Experiment on Public Speaking Anxiety in Response to Three Different Types of Virtual Audience," In Presence: Teleoperators and Virtual Environments, Vol. 11, no. 1, pp. 68 - 78, February 2002.

[12] M.M. North, S.M. North, and J.R. Coble, "Virtual Environment Psychotherapy: A Case Study of Fear of Flying Disorder," In Presence: Teleoperators and Virtual Environments, Vol. 6, no. 1, pp. 127 - 132, February 1997.

[13] A. Rizzo, "From training to toy to treatment: Design and development of a PTSD VR Therapy application for Iraq war veterans," Proc. of the Third International Workshop on Virtual Rehabilitation (IWVR'04), EPFL, Lausanne, Switzerland, 2004.

[14] F. Alcantud, G. Herrera, G. Labajo, I. Dolz, C. Gayá, V. Avila, A. Blanquer, J.L. Cuesta, and J. Arnáiz, "Assessing Virtual Reality as a Tool for Support Imagination," Proceedings of Computer Helping People with Special Needs: 8th International Conference, ICCHP 2002, Linz, Austria, July 15-20, 2002.

[15] N. Parés, A. Carreras, J. Durany, J. Ferrer, P. Freixa, D. Gomez, O. Kruglanski, R. Parés, J.I. Ribas, M. Soler, and A. Sanjurjo, "MEDIATE: An interactive multisensory environment for children with severe autism and no verbal communication," Proc. of the Third International Workshop on Virtual Rehabilitation (IWVR'04), EPFL, Lausanne, Switzerland. (Awarded the Biodex Best Paper Award), pp 43-52, 2004.

[16] P.L. (Tamar) Weiss, P. Bialik, and R. Kizony, “Virtual Reality Provides Leisure Time Opportunities for Young Adults with Physical and Intellectual Disabilities," Cyberpsychology \& Behavior, Vol. 6, no. 3, pp. 335-342, 2003.

[17] D. Rand, R. Kizon, and P.L. Weiss, "Virtual reality rehabilitation for all: Vivid GX versus Sony PlayStation II EyeToy," Proc. 5th Intl Conf. Disability, Virtual Reality \& Assoc. Tech., Oxford, UK, pp. 87-94, 2004.

[18] S. Parsons, and P. Mitchell, "The potential of Virtual Reality in social skills training for people with autistic spectrum disorders". Journal of Disability Research 46(5) pp. 430-443, 2002.

[19] B. Robins, P. Dickerson, P. Stribling, and K. Dautenhahn, "Robotmediated joint attention in children with autism: A case study in robot-human interaction," Interaction Studies 5:2, pp. 161-198, 2004

[20] B. Robins, K. Dautenhahn, R. te Boekhorst, and A. Billard, “Effects of repeated exposure to a humanoid robot on children with autism," In S. Keates, J. Clarkson, P. Langdon and P. Robinson (Eds.) Designing a More Inclusive World, Springer Verlag, London, pp. 225-236, 2004.

[21] K. Dautenhahn, I. Werry, "Towards Interactive Robots in Autism Therapy: Background, Motivation and Challenges," Pragmatics and Cognition 12(1), pp. 1-35, 2004.

[22] H. Timmermans, G. van Wolferen, P. Newland, and S. Kunath, "MEDIATE: Key Sonic Developments in an Interactive Installation for Children with Autism," ICMC 2004, University of Miami, USA, 2004.

[23] N. Parés, A. Carreras, and M. Soler, "Non-invasive attitude detection for full-body interaction in MEDIATE, a multisensory interactive environment for children with autism," Proc. of Vision, Modeling, and Visualization 2004 (VMV'04), Stanford, California, USA, pp. 37-46, 2004.

[24] F. Restle, "Theory of serial pattern learning: Structural Trees," Psychological review, American Psychological Association, Washington, DC, no. 77, pp. 454-495, 1970.

[25] R. de Groot, "Flexibility; pitch organisation in recent works by 
Theo Loevendie," Keynotes 14, 1981/2, DONEMUS Amsterdam, Holland, 1981.

[26] MEDIATE EC Final Report, URL:http:/ / www.port.ac.uk/research/mediate/deliverables/fil es/filetodownload,24165,en.pdf , last accessed 27/02/05.

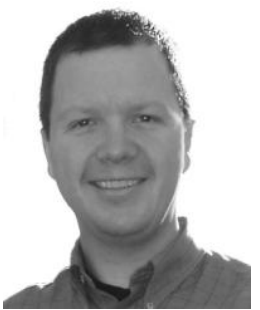

Narcís Parés received his BSc in Computer Engineering by Polytechnic University of CataIonia, Barcelona, Spain in 1991, an MSc in CG, Al \& Image Processing by Autonomous University of Barcelona in 1993 and a PhD in Audiovisual Communication specialized on Virtual Reality by Universitat Pompeu Fabra (UPF), Barcelona in 2001. He has been assistant professor at the Computer Science Unit, Autonomous University of Barcelona, associate professor at Audio-visual Communication Department, UPF and is currently full time assistant professor in the Department of Technology, UPF. Has received the Biodex Best Paper award at IWVR'04. His research interests are in Interactive Communication, specifically interaction with real time generated stimuli \& VR.

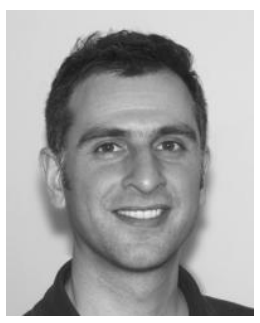

Paul Masri received his BEng in Electronic Engineering (University of Bristol, UK) in 1991. His PhD entitled "Computer Modelling of Sound for Transformation and Synthesis of Musical Signals" was awarded in 1997 by Uni. Bristol. He currently owns and runs a web development business (www.worldwithoutwalls.co.uk) but enjoys remaining an active member of the research community. In 2000, he co-created (with Joel Laird) a permanent, interactive exhibit at the London Science Museum demonstrating physical modeling of drums. His research interests are interactive devices and systems for the creative arts.

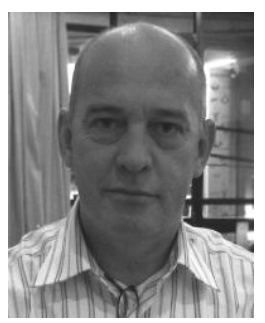

Gerard van Wolferen graduated in Musical Composition with Theo Loevendie at the Rotterdam Conservatorium in 1981. In 1988 he received the doctoraal (sic) degree in Musicology at the Universiteit van Amsterdam. Specializations: theoretical musicology and ethnomusicology. Thesis: Musical Structure and Perception. Currently teacher of analysis \& synthesis of music, ethnomusicology and composition at the faculty of Art, Media \& Technology, of the Utrecht School of the Arts since 1991; Head of the Faculty Department of Music \& Technology; Researcher at URIDC (Utrecht Research Institute of Digital Culture) with an interest in musical structure, cognition, computational analysis and in projects for people with special needs.

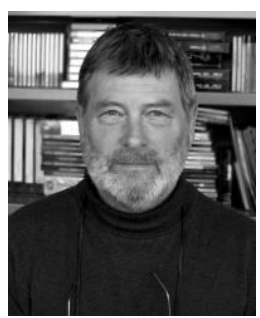

Chris Creed received his BSc in Psychology (University of Hull, UK) in 1965 and his PhD (Social Psychology) in 1972 (University of Southampton, UK). He has worked as a researcher and lecturer at the Institute of Education, London University; The University of Southampton; The University of Aberdeen; and currently at the University of Portsmouth, UK. Previous design and research involved building a simulator for training senior Fire Officers in command and control, as well as producing interactive programmes for architects and for medical students. For the last three years he has been the overall coordinator of the MEDIATE project. His research interests concern user behavior in responsive environments. 